\title{
Pregnant Patients with Deep Venous Thrombosis: Management and the Incidence of Thrombophilic Risk Factors
}

\section{Ekim $\mathrm{M}^{*}$ and Ekim $\mathrm{H}^{2}$}

${ }^{1}$ Department of Biochemistry, Bozok University School of Health, Yozgat, Turkey

${ }^{2}$ Department of Cardiovascular Surgery, Bozok University School of Medicine, Yozgat, Turkey

*Corresponding author: Ekim M, MD, Department of Biochemistry, Bozok University School of Health, Yozgat, Turkey, E-mail: meralekim@yahoo.com

Citation: Ekim M, Ekim H (2015) Pregnant Patients with Deep Venous Thrombosis: Management and the Incidence of Thrombophilic Risk Factors. J Anesth Pati Care 1(1): 102. doi: 10.15744/2456-5490.1.102

Received Date: March 24, 2015 Accepted Date: June 25, 2015 Published Date: June 26, 2015

\begin{abstract}
Objective: There is lack of knowledge about anticoagulant therapy in pregnancy and an acceptable guide for their use is based on case series and the opinion of scientists. For this reason, we carried out a study on pregnant women suffering from acute deep venous thrombosis (DVT) using enoxaparin twice daily dose. Additionally, all patients were screened for hypercoagulopathy.

Methods: This study was performed in 30 pregnant women with acute DVT who managed between January 2007 and September 2012 in Dursun Odabas Medical Center, Van, Turkey. In all patients, the diagnosis of DVT was based upon on Wells scoring system and D-dimer testing and confirmed by venous Doppler ultrasonography. Blood samples were collected to determine factor V Leiden (FVL), prothrombin gene mutation (PT G20210A). In all patients, enoxaparin therapy was administered. The initial dose of enoxaparin was administered with $1 \mathrm{mg} / \mathrm{kg}$ twice daily and adjusted for weight changes throughout pregnancy.

Results: In this series, the age range was $21-40$ years with an average of $26 \pm 11$ years. No allergic reactions were observed during pregnancy in any of the patients. Heterozygous FVL mutation was observed in six (20\%) patients, and heterozygous PT G20210A mutation in one (3.3\%). None of the patients were observed to have pulmonary embolism, massive bleeding, or thrombocytopenia. Nineteen patients showed successful re-canalization. There were no clinical features of post thrombotic syndrome during follow up period in all patients.

Conclusion: In this series, twice-daily enoxaparin therapy was found to be efficient and safe for mothers and their babies and was promising about a better quality of life for the pregnant patients. Among the pregnant patients with DVT, FVL mutation is not rare. Therefore, pregnant patients with DVT may be screened for inherited risk factors. We suggest that monitoring of enoxaparin concentration may not be necessary in pregnant patients without thrombophilia. However, further studies should be required.

KeyWords: Deep Vein Thrombosis; Thrombophilia; Enoxaparin
\end{abstract}

\section{Introduction}

Deep vein thrombosis (DVT) and pulmonary embolism (PE) are considered to be different variants of the same disease, and both have accepted venous thromboembolism (VTE) [1]. Pregnancy carries a high risk of thromboembolism [2].

Venous flow stasis is more frequently encountered risk factor in pregnant women due to hormonal alterations [3]. Also there are additional risk factors such as thrombophilia, cardiac disease, sickle cell anemia, lupus, overweight, anemia, diabetes mellitus, increased arterial tension, and tobacco use [4]. The increased risk factors of thrombogenic phenomena should be regarded as protective for pregnant women against excessive bleeding during miscarriage or birth $[3,5]$. But thromboembolism in pregnancy can be life-threatening for both the mother and the fetus [6].

Increased plasma concentrations of coagulation factors and fibrinolysis inhibitors, alterations in venous flow and changes within vascular walls are usually associated with pregnancy [7]. Therefore, the risk of pregnancy related VTE is five times higher in pregnant women compared to non- pregnant women of same age group [8].

Current guidelines recommend therapy with adjusted-dose subcutaneous low molecular weight heparin (LMWH) over adjusteddose unfractionated heparin (UFH) for pregnant women with acute DVT [9]. However, there is no large volume studies on anticoagulants treatment in pregnancy and guidelines for their use are based on case series and the opinion of physicians [4,10]. For this reason, we report our experience on pregnant women suffering from acute DVT using enoxaparine twice daily dose. Also all patients were screened for hypercoagulopathy.

\section{Methods}

This study was performed in 30 pregnant women with acute DVT who managed between January 2007 and September 2012 in Dursun Odabas Medical Center, Van, Turkey. The study was performed in accordance with the regulations of the ethics committee of Bozok University and the Declaration of Helsinki. The informed consent was obtained from every participating patient after 
the aim of the study had been fully elucidated. In all patients, the diagnosis of DVT was based upon on Wells scoring system and D-dimer testing and confirmed by venous Doppler ultrasonography. D-dimer concentrations were provided in micrograms per liter $(\mu \mathrm{g} / \mathrm{L})$ and considered negative if less than $500 \mu \mathrm{g} / \mathrm{L}$. All patients had pain and increased diameter of the affected limb. Patients with renal disease or with phlegmasia cerulean dolens were excluded from the study. Routine biochemical blood tests, liver function tests, blood counts, electrocardiogram and chest radiograph were performed in all patients. Blood samples were collected to determine factor V Leiden (FVL), prothrombin gene mutation (PT G20210A).

In all patients, enoxaparin therapy was administered. The initial dose of enoxaparine was administered with $1 \mathrm{mg} / \mathrm{kg}$ twice daily and adjusted for weight changes throughout pregnancy. Lower limb elevation and compression stockings were used and mobility was not restricted. Antifactor Xa activity was not measured routinely during study. Peak antifactor Xa level was measured 4 hours after injection at least once during treatment to evaluate anticoagulation efficacy in patients with thrombophilia. The patients were discharged to home after one week if they responded well to treatment and if their complaints ameliorated. Anticoagulant therapy was sustained until delivery in all patients and at least a 12-hour period was ensured between the last dose and delivery. The decision on mode of delivery was the responsibility of the department of gynecology. LMWH treatment was reinstituted 12 hours after delivery. Pneumatic compression devices were used until LMWH therapy was restarted. Oral warfarin was overlapped to LMWH therapy at the first or second days of postpartum. LMWH and coumadin therapy was sustained for at least five days. Thereafter, LMWH administration was discontinued, provided that INR had been at a therapeutic level (2-3) for at least 2 consecutive days. Warfarin treatment was continued until completion of the anticoagulant therapy. Breastfeeding was advised for all patients even during oral anticoagulant treatment. All patients were regularly monitored with INR value to avoid complications during the warfarin therapy.

The efficiency of anticoagulant treatment was controlled in the vascular surgery department by circumferential measurements of the affected limbs and by venous Doppler ultrasonography examination. The circumference of the limb was measured at the midthigh and mid-calf levels. The patients with pulmonary embolism suspicion were consulted by the specialist of Chest Diseases Department. Thrombocyte counts and hemorrhagic complications of the patients were recorded during the anticoagulant treatment. All patients were followed up 18 months after complete course of anticoagulation. During follow up period, the patients were assessed according to the CEAP classification.

\section{Results}

In this series, the age range was 21 - 40 years with an average of $26 \pm 11$ years. No allergic reactions were observed during pregnancy in any of the patients. Also, plasma-creatinine concentrations were within the normal range during pregnancy in all patients.

D-dimer levels were initially found to be positive in all patients. Thrombosis panel study revealed heterozygous FVL in six (20\%) patients, and heterozygous PT G20210A mutation in one (3.3\%). The mean antifactor Xa levels $(0.61 \pm 0.11 \mathrm{IU} / \mathrm{ml})$ were within the effective range for patients with thrombophilia (normal range: 0.4-1.2 IU/ml).

Six patients presented with DVT in the first trimester, 11 patients in the second trimester while 23 patients represented in the third trimester. A total of 18 patients were multipara. DVT was diagnosed in the left lower extremity in 25 patients. Venous Doppler ultrasonography revealed that 22 patients had iliofemoral venous segment involvement (Table 1).

\begin{tabular}{|c|c|}
\hline Level of thrombosis & No. of patients and (\%) \\
\hline İliofemoral & $22(73.3 \%)$ \\
\hline Femoropopliteal & $4(13.3 \%)$ \\
\hline Femoral & $3(10 \%)$ \\
\hline Popliteal & $1(3.3 \%)$ \\
\hline
\end{tabular}

None of the patients was observed to have pulmonary embolism or thrombocytopenia. During treatment, minor bleeding (less than $200 \mathrm{~mL}$ ) developed in four patients, of whom two had vaginal bleeding, one had hematuria, and one had epistaxis. In two patients with vaginal bleeding and one patient with hematuria, clinical findings were improved after decreasing the dosage of enoxaparine. The woman with epistaxis healed spontaneously. No miscarriage occurred during the pregnancy. Spontaneous vaginal delivery occurred in 25 patients, and cesarean section was required in five. All women had healthy full-borne babies. No complication related with post-delivery anticoagulation treatment was occurred in the mothers and neonates.

Venous ultrasonography was repeated at intervals of 6-8 weeks. Nineteen patients showed successful re-canalization. Clinical features of post thrombotic syndrome were not observed during follow up period in all patients.

\section{Discussion}

Currently diagnostic imaging algorithms for the pregnant patients with DVT are usually extrapolated from the studies of the nonpregnant women. This presumes that demographics, pathophysiological characteristics of DVT in pregnant patients are similar to those in non-pregnant women [11]. 
UFH or LMWH cannot cross the placental barrier and they are given safely during pregnancy period [3]. Compared with UFH, LMWHs have higher bioavailability, lower rates of adverse effects, including thrombocytopenia, osteoporosis, hemorrhage, and allergic reactions $[12,13]$. UFH also has a short half-life, and dose adjustment is necessary, adjusting according to the activated partial thromboplastin time (aPTT) level. Additionally, aPTT levels measured during heparin treatment may not be very reliable in pregnant women due to heparin resistance. The resistance is explained by elevated fibrinogen, factor VIII and heparin-binding protein levels, which in turn leads to higher doses of heparin infusion in pregnant patients, resulting in an increased risk of bleeding [5].Thus, LMWHs are preferred for anticoagulation during pregnancy.

Current recommendations for the duration of anticoagulation treatment in pregnancy range from three to six months, including six weeks postpartum. This treatment duration should be longer than one year for women with VTE and antiphospholipid antibody syndrome and for women with two or more thrombophilic risk factors [14].

A cesarean operation should not be performed while the patient is in a fully anticoagulated condition; this can lead to uncontrolled hemorrhage and maternal death [15]. When delivery is predictable, as for elective induction or planned cesarean delivery, heparin or LMWH should be discontinued 24 hours before delivery [14]. To minimize hemorrhagic complications, resumption of anticoagulation should be delayed until 12 hours after vaginal delivery, 12 hours after epidural catheter removal, or 24 hours cesarean section. The post-trombotic syndrome may develop in up to $60 \%$ of patients with DVT [15].

FVL and the PT G20210A each confer approximately a 10-fold increased risk for VTE in pregnancy. Combined they confer an increased risk of approximately 70 -fold, which is similar to the approximately 80 -fold increased risk associated with homozygosity of FVL [3].

Warfarin crosses the placental barrier and may lead to miscarriage, stillbirth, congenital anomalies, fetal bleeding, and poor neurological outcome [3]. It affects five percent of fetuses that are exposed to the drug between six and nine weeks of pregnancy [15]. However, it is not secreted in breast milk in clinically significant levels [5]. Therefore, warfarin treatment was not used during pregnancy in our series; while it has used in the period of breast-feeding after delivery.

In the nonpregnant patient, LMWH is usually injected once daily with the use of a weight-adjusted dose regimen [15]. Pharmacokinetic studies showed that enoxaparin had a shorter half-life in pregnant patients with DVT. The shorter half-life may be explained by the higher glomerular filtration rate, greater volume of distribution, or placental heparinase that accompanies pregnancy [16]. Therefore, we have preferred twice daily injection of LMWH in pregnant patients.

When DVT develops during pregnancy, it is more likely to be proximal, large, and in the left lower limb [2]. While DVT incidence in the lower left limb is $55 \%$ under normal conditions, this incidence rises to $80 \%-90 \%$ during gestation [5]. This left lower limb predominance is thought to be attributable to a relative stenosis of the left common iliac vein where it lies between the lumbar vertebral body and the right common iliac artery, but the real reason is unknown [2,17]. Likewise, while DVT incidence in the iliofemoral region for non-pregnant women is $9 \%$, it is $70 \%$ for pregnant patients [18]. In this series, incidence of DVT in iliofemoral region was found to be $73.3 \%$, similar to previous studies.

Thrombophilia is present in 20-50 \% of women who have VTE during gestation and puerperium. Both hereditary and acquired thrombophilia increase the risk of VTE [19]. Inherited thrombophilia includes FVL, PT G20210A and congenital deficiencies of antithrombin, protein $\mathrm{C}$ and protein S [20]. Although monitoring antifactor Xa level during LMWH treatment is not routinely required, measuring peak antifactor Xa level 3 hours after administration is suggested in pregnant patients, especially in cases with thrombophilia [5]. Additionally, anti-Xa levels should be monitored if there are complications such as bleeding or incorrect dosage [21].

Theoretically, fibrinolysis appears favorably in the management of VTE. However, the risk of severe hemorrhage precludes its use during pregnancy [22]. Although new endovascular treatment modalities have been available, the large majority of pregnant patients still continue to undergo anticoagulation as the sole treatment [23].

Although anticoagulant treatment using LMWHs seems to be effective and safe in pregnant patients, optimal dosing regimens are still uncertain [24]. Enoxaparin is the most frequently injected LMWH in pregnant women. Nonpregnant women with DVT can usually be treated as outpatients, but pregnant patients, who tend to have large thrombi, are usually hospitalized [10]. Pregnant patients with VTE should be managed for a minimum of 3-6 months, as in nonpregnant counterparts. However, anticoagulation should be sustained from the diagnosis of the DVT, through delivery and for 6 weeks after delivery [20].

About 2\% of pregnancy-related DVT develops in the upper limb [25]. But the cases of DVT developing in association with reproductive technologies occur predominantly in the upper limb or neck in pregnant patients who had ovarian hyperstimulation syndrome [17].

UFH carries low bleeding risk at the time of delivery. Anticoagulation treatment may be converted from LMWH to UFH in the last month of pregnancy or if birth will be soon. Additionally heparin has low spinal or epidural bleeding risk with regional anesthesia [17]. In our series, although enoxaparin therapy was not converted to UFH treatment, we had not encountered any complications at the time of delivery. 


\section{Conclusion}

In this series, twice-daily enoxaparin therapy was found to be efficient and safe for mothers and their babies and was associated with an obvious improvement in quality of life for the pregnant patients with minimal stay in hospital by adopting a multidisciplinary approach. Among the pregnant patients with DVT, FVL mutation is not rare. Therefore, pregnant patients with DVT may be screened for inherited risk factors. We suggest that monitoring of enoxaparin concentration may not be necessary in pregnant patients without thrombophilia. However, further studies should be required.

\section{References}

1. Sakuma M, Nakamura M, Yamada N, Ota S, Shirato K, et al. (2009) Venous thromboembolism: deep vein thrombosis with pulmonary embolism, deep vein thrombosis alone, and pulmonary embolism alone. Circ J 73: 305-9.

2. James AH (2009) Venous thromboembolism: mechanisms, treatment, and public awareness: Venous thromboembolism in pregnancy. Thromb Vasc Biol 29: 326-31.

3. James AH (2007) Prevention and management of venous thromboembolism in pregnancy. Am J Med 120: S26-34.

4. James AH (2010) Pregnancy and thrombotic risk. Crit Care Med 38: S57-63.

5. Narin C, Reyhanoglu H, Tülek B, Onoglu R, Ege E, et al. (2008) Comparison of different dose regimen of enoxaparin in deep vein thrombosis therapy in pregnancy. Adv Ther 25: 585-94.

6. Akbas F, Oral E, Bircan R, Erzik C, Cirakoglu B, et al. (2003) The effect of hereditary thrombophilia on some pregnancy complications. T Klin J Gynecol Obst 13: $106-12$.

7. Brown HL, Hiett AK (2010) Deep venous thrombosis and pulmonary embolism in pregnancy: diagnosis, complications, and management. Clin Obstet Gynecol 53: 345-59.

8. Soomro RM, Bucur IJ, Noorani S (2002) Cumulative incidence of venous thromboembolism during pregnancy and puerperium: a hospital-based study. Angiology 53: 429-34.

9. Bates SM, Greer IA, Middeldorp S, Veenstra DL, Prabulos AM, et al. (2012) VTE, thrombophilia, antithrombotic therapy, and pregnancy: Antithrombotic Therapy and Prevention of Thrombosis, 9th ed: American College of Chest Physicians Evidence-Based Clinical Practice Guidelines. Chest 141: e691S-736S.

10. James AH (2012) Prevention and treatment of venous thromboembolism in pregnancy. Clin Obstet Gynecol 55: 774-87.

11. Chan WS, Spencer FA, Ginsberg JS (2010) Anatomic distribution of deep vein thrombosis in pregnancy. CMAJ 182: 657-60.

12. Greer IA, Nelson-Piercy C (2005) Low-molecular-weight heparins for thromboprophylaxis and treatment of venous thromboembolism in pregnancy: a systematic review of safety and efficacy. Blood 106: 401-7.

13. Lepercq J, Conard J, Borel-Derlon A, Darmon JY, Boudignat O, et al. (2001) Venous thromboembolism during pregnancy: a retrospective study of enoxaparin safety in 624 pregnancies. BJOG 108: 1134-40.

14. Dresang LT, Fontaine P, Leeman L, King VJ (2008) Venous thromboembolism during pregnancy. Am Fam Physician 77: 1709-16.

15. Marik PE, Plante LA (2008) Venous thromboembolic disease and pregnancy. N Engl J Med 359: 2025-33.

16. Barbour LA, Oja JL, Schultz LK (2004) A prospective trial that demonstrates that dalteparin requirements increase in pregnancy to maintain therapeutic levels of anticoagulation. Am J Obstet Gynecol 191: 1024-9.

17. James AH (2009) Pregnancy-associated thrombosis. Hematology Am Soc Hematol Educ Program doi: 10.1182/asheducation-2009.1.27.

18. Eldor A (2002) The use of low-molecular-weight heparin for the management of venous thromboembolism in pregnancy. Eur J Obstet Gynecol Reprod Biol 104: 3-13.

19. James AH (2008) Thromboembolism in pregnancy: recurrence risks, prevention and management. Curr Opin Obstet Gynecol 20: 550-6.

20. Fogerty AE, Connors JM (2009) Management of inherited thrombophilia in pregnancy. Curr Opin Endocrinol Diabetes Obes 16: 464-9.

21. Voke J, Keidan J, Pavord S, Spencer NH, Hunt BJ, et al. (2007) The management of antenatal venous thromboembolism in the UK and Ireland: a prospective multicentre observational survey. Br J Haematol 139: 545-58.

22. Pillny M, Sandmann W, Luther B, Müller BT, Tutschek B, et al. (2003) Deep venous thrombosis during pregnancy and after delivery: indications for and results of thrombectomy. J Vasc Surg 37: 528-32.

23. McLafferty RB (2008) Endovascular management of deep venous thrombosis. Perspect Vasc Surg EndovascTher 20: 87-91.

24. Romualdi E, Dentali F, Rancan E, Squizzato A, Steidl L, et al. (2013) Anticoagulant therapy for venous thromboembolism during pregnancy: a systematic review and a meta-analysis of the literature. J Thromb Haemost 11: 270-81.

25. James AH, Tapson VF, Goldhaber SZ (2005) Thrombosis during pregnancy and the postpartum period. Am J Obstet Gynecol 193: 216-9.

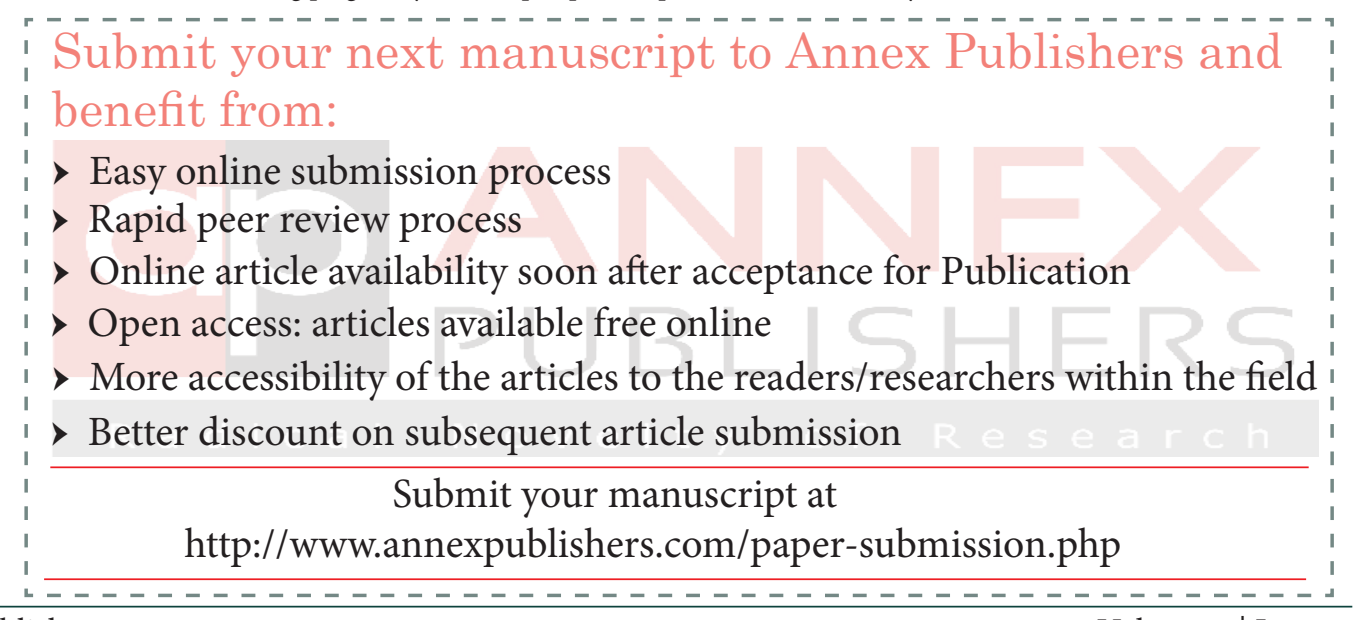

